\title{
E-learning in GBIF Capacity Activities
}

\author{
Katia Cezón García ${ }^{\ddagger}$, Cristina Villaverde ${ }^{\ddagger}$, Carmen Lujano ${ }^{\ddagger}$, Laura Russell ${ }^{\S}$ \\ ‡ Royal Botanic Garden - CSIC - GBIF, Madrid, Spain \\ $\S \mathrm{GBIF}$, Copenhagen, Denmark
}

Corresponding author: Katia Cezón García (katia.gbif@gmail.com), Cristina Villaverde (cvillaverde@rib.csic.es)

Received: 11 Jun 2019 | Published: 18 Jun 2019

Citation: García K, Villaverde C, Lujano C, Russell L (2019) E-learning in GBIF Capacity Activities. Biodiversity Information Science and Standards 3: e37070. https://doi.org/10.3897/biss.3.37070

\begin{abstract}
E-learning is a training method increasingly used as it enhances opportunities for participation, allows active tracking of courses and enables wider flexibility for timetables and organization. The implementation of an e-learning platform managed by GBIF Spain was aimed at exploring other ways of creating capacity, reaching a broader audience and multiplying the impact of a well-consolidated training facility. The first platform, used by the Spanish node in 2012, was the open source tool ATutor, which was migrated to Chamilo in 2017 and is available at http://elearning.gbif.es. Since then, these platforms have hosted courses focused on biodiversity data mobilization and data use, addressing both users and data publishers. GBIF.es offers the platform to other organizations in the community so the entire GBIF network can benefit from this service, taking advantage of the already invested resources. Additionally, the GBIF Secretariat uses the platform for developing training activities in the Biodiversity Information for Development (BID) and Biodiversity Information for Asia (BIFA) programmes reaching participants in sub-Saharan Africa, the Caribbean and the Pacific, and has implemented the Biodiversity Data Mobilization course for use in other programmes such as Biodiversity Data Management Skills for Students (BioDATA) and Biodiversity Information Management and Reporting (BIMR), reaching communities in Eurasia and South-East Europe respectively. GBIF Argentina has also used it to offer a course on biodiversity data quality and publication. In this session, we will overview the GBIF.es e-learning platform, highlight some successful use cases of the virtual training tool and show how it helped GBIF Spain reach a broader community. We will also present plans for future expansion.
\end{abstract}




\section{Keywords}

e-learning, GBIF, biodiversity data, Chamilo, capacity enhancement

\section{Presenting author}

Cristina Villaverde

\section{Presented at}

Biodiversity_Next 2019 